\title{
THE EFFECT OF KNOWLEDGE, BEHAVORS, AND ATTITUDES TOWARDS DIETARY FATTY ACIDS ON BLOOD LIPID LEVELS
}

\author{
Amber Deckard
}




\section{CHAPTER 1 INTRODUCTION}

Americans are currently experiencing an obesity epidemic with an estimated $70.7 \%$ of adults over the age of twenty finding themselves in the overweight or obese classifications. Researchers predict that by the year 2030, approximately 50\% of the United States (U.S.) population will be classified as obese. Continuously growing at an alarming rate, the obesity epidemic is not projected to stop any time soont.

Obesity is a disease that is oftenaccompanied by other comorbidities including, but not limited to: insulin resistance, Type II Diabetes, inflammatory conditions, cardiovascular disease (CVD), cancer, and decreased longevity. Each of these diseases can be assuaged through lifestyle changes and nutrition intervention; in fact, the number one leading cause of death in the U.S. is CVD which is directly related to weight and is preventable. A large contributor to CVD is plaque buildup caused by deposits of fatty acids in the arteries. Over time, diets high in fat will continue to accumulate plaque until a clot forms leading to serious and fatal conditions. Many research studies have examined the impact of various dietary fatty acids on CVD and overall health, including looking into how lifestyle factors including smoking, lack of physical activity, excessive sodium and alcohol intake directly impact CVD2. Research is lacking on how knowledge and education of dietary fatty acids can affect blood lipid levels.

The purpose of this this research project was two-fold. We aimed to determine 1) knowledge, attitudes, and behaviors of dietary fats using the modified General Nutrition Knowledge Questionnaire (GNKQ); and 2) correlations between anthropometric data, GNKQ responses and blood lipid levels. We hypothesize there will be an inverse relationship between knowledge of dietary lipids as a whole and the blood lipid levels compared to the standard ranges. We hypothesize that the more knowledge an individual has on about dietary fats, the lower their adverse blood lipids will be.

\section{CHAPTER II LITERATURE REVIEW}

\section{Chronic Disease}

In the U.S., two in every three adults are classified as being overweight or obese. This is a key factor into health and disease prevention, because individuals falling into this weight classification are at greater risk for insulin resistance, 
type II diabetes, hypertension, inflammatory conditions, and various other diseases/conditions3. These conditions are often comorbidities and accompany other preexisting medical diagnosis or will escalate into others.

For example, through lack of treatment or control what was originally diagnosed as hypertension can develop into CVD. There is a strong relationship across international guidelines in regarding the importance of exercise, weight balance, cessation of smoking, monitoring hypertension, and optimal lipid profile as precautions to prevent CVD4. One reason for a lack of optimal blood lipid levels and this development into CVD may be a diet high in fatty acids.

\section{Dietary Fatty Acids}

Dietary fatty acids (FA) are essential for survival and are a key component of the diet; however, too much consumption is linked to obesity and CVD7. Due to the risk of excess FA accumulating in the body and leading to other co-morbidities, the breaking down and oxidation of fat in the body is beneficial and prevents accumulation from occurring. Weight gain occurs when fat accumulates in the form of triacylglycerols in adipocytes. Due to this accumulation of fat, health complications associated with excess weight can occur.

There are four different types of FAs found in varying quantities in different types of fats: saturated fatty acids (SFA), polyunsaturated fatty acids (PUFA), monounsaturated fatty acids (MUFA), and trans fatty acids (TFA). Each of these fats alter the cholesterol levels in the body differently.

\section{Cholesterol Trends}

An increase in SFA in the diet increases the LDL cholesterol and the total blood cholesterol levels as well. A high PUFA diet has a reducing effect on LDL, HDL, total cholesterol, and triglyceride levels. A high MUFA diet has the most benefits with it lowering LDL levels and total cholesterol without lowering the HDL levels. A diet high in TFA increases LDL and total cholesterol levels8.

Researchers have determined PUFA rich meals, as discussed earlier, to be beneficial to overall health and disease prevention. The researchers further have found that when an individual consumes a PUFA rich diet, it initiates fat oxidation to a greater extent after eating the occasional high SFA meal versus a control diet in which PUFA levels are low, approximately seven percent, as compared to the PUFA rich diet which is 21\%9. PUFA trigger the oxidation of the saturated fat to occur at a higher extent, breaking them down more efficiently and effectively. This is beneficial for health because when saturated fats accumulate in the body this is what leads to high LDL content and unhealthy cholesterol tendencies as discussed earlier.

Diets high in the certain fatty acid means, for this paper, diets exceeding the daily recommendations of those listed in Table 1. 


\section{Table 1: FATTY ACID'S EFFECT ON BLOOD LIPID LEVELS}

\begin{tabular}{ll}
\hline \multicolumn{1}{c}{ Fatty Acid } & \multicolumn{1}{c}{ Effect } \\
\hline SFA & $\begin{array}{l}\text { Increases total cholesterol } \\
\text { Increases LDL cholesterol }\end{array}$ \\
\hline & Decreases total cholesterol \\
PUFA & Decreases LDL cholesterol \\
& Decreases HDL cholesterol \\
\hline MUFA & Decreases total cholesterol \\
& Decreases LDL cholesterol \\
TFA & Increases total cholesterol \\
& Increases LDL cholesterol \\
\hline *Saturated Fatty Acids (SFA), Polyunsaturated Fatty Acids (PUFA), \\
Monounsaturated Fatty Acids (MUFA), and Trans Fatty Acids (TFA). \\
**Low Density Lipoprotein (LDL) also known as "bad cholesterol", High \\
Density Lipoprotein (HDL) also known as "good cholesterol".
\end{tabular}

\section{Table 2: RECOMMENDED INTAKE OF FATTY ACIDS}

\begin{tabular}{lc}
\hline \multicolumn{1}{l}{ Recommended Dietary Intake (\% of total kcal) } \\
\hline SFA & $<10 \%$ \\
\hline MUFA & $13 \%-18 \%$ \\
\hline PUFA & $6 \%-11 \%$ \\
\hline TFA & $<1 \%$ \\
\hline *Saturated fatty acids (SFA), Polyunsaturated fatty acids (PUFA), \\
Monounsaturated fatty acids (MUFA), and Trans fatty acids (TFA). \\
\hline
\end{tabular}




\section{Lipid Panel}

The Mayo Clinic defines cholesterol as the waxy substance found in fats in the blood that can be deposited in blood vessels and hardening leading to atherosclerosis and many other chronic diseases discussed in Chronic Diseases5. Cholesterol levels are determined through a blood lipid panel blood test which looks at the individual components of cholesterol: Total cholesterol, low-density lipoprotein (LDL), high-density lipoprotein (HDL), triglyceride levels, and a risk ratio (LDL/HDL in ratio format). The optimal levels are stated in Table 36. Optimal levels have been established based on research done on where atherosclerosis and other chronic diseases begin to occur; ranges are based on NCEP guidelines and are used for treatment and diagnosis at all medical clinics and research facilities including the Mayo Clinic stated prior.

According to the Mayo Clinic, high cholesterol is an asymptomatic condition and will develop into plaque deposits, atherosclerosis, and eventually CVD. Due to the lack of symptoms, it is important to have annual doctor visits that include a lipid panel and efforts to maintain those optimal levels5.

\section{Table 3: OPTIMAL LIPID PANEL LEVELS}

\begin{tabular}{lc}
\hline & Normal Ranges \\
\hline Total cholesterol & $<200 \mathrm{mg} / \mathrm{dL}$ \\
\hline LDL cholesterol & $<150 \mathrm{mg} / \mathrm{dL}$ \\
\hline HDL cholesterol & $>39 \mathrm{mg} / \mathrm{dL}$ \\
\hline Triglycerides & $<100 \mathrm{mg} / \mathrm{dL}$ \\
\hline Risk Ratio & $<3.22$ \\
\hline
\end{tabular}

\section{Education}

Recently, there has been an interest in how education and income levels affect a person's health. Researchers have found that larger incomes correlate to lower likeliness of disease and premature death. Higher incomes pair with lower risk of disease, and lower incomes with increased risk of disease, both representing an inverse relationship between income and disease diagnosis17. considering income as a factor in health sheds new light for public 
health fields and nutrition education. When looking at nutrition education though, little research has been done. In Europe, the "Think Again About Cholesterol Survey" helped shed some light on the lack of nutrition education. The survey found that one in four adults believed that cholesterol does not need to be a concern until someone shows signs or symptoms, and $92 \%$ of participants did not know their own LDL levels or had never had their cholesterol levels tested12. This survey, though beneficial, still did not look at how knowledge of diet relates to health.

In another study in northern Italy, researchers looked at how teachers could potentially help prevent CVD. Five teachers received Primary Prevention of Adult Cardiovascular Disease education (PP-CVD). The PP-CVD education consisted of multiple readings regarding information on atherosclerosis, risk factors, preventative measures, and diet impact with emphasis on adolescence and the young-adult population. One booklet in particular written by Roberto Aquilani, one of the lead researchers on the study, was chosen by the researchers for the five teachers to adopt into their preexisting biology curriculum. The booklet offered education on risk factors, impact, and ways to improve blood lipid levels through the diet. Teachers then went about their usual schedules teaching thirteen classes at the high school, with the modified biology curriculum containing nutrition education. After a six-month period, compared to the control group, the teachers who had been educated had students who improved their lipid profile consequently to autonomous changes in dietary habits; this was seen through multiple choice questions for comprehension of material, anthropometric data, food diaries, and blood values. More than 70\% of students had both a reduction of $>10 \%$ in LDL cholesterol and an increase of $>7 \%$ in HDL cholesterol. Students additionally in the PP-CVD educated classrooms reported less snacks and sugary beverages per week then those in the control.13 This survey shows the relationship in education over CVD prevention and lipid profiles. However, when students do not receive any education, the question remains; how does present knowledge on nutrition overall and specific nutrients effect lipid panel? More research is needed to relate education of a specific nutrient to health as in how the knowledge of fats affect an individual's lipid panel. 


\section{CHAPTER III METHODS}

\section{Study Design}

The TCU Institutional Review Board approved this cross-sectional designed study that utilized a modified GNKQ questionnaire. The questionnaire originally contained over 200 questions discussing general nutrition knowledge and all components of the diet: vitamins/minerals, carbohydrates, proteins, and fats. All questions not pertaining to dietary fatty acids were excluded from the questionnaire. The modified GNKQ was then entered into Qualtrics. Participants could choose to either complete the online questionnaire via Qualtrics only, or to complete the questionnaire and participate in a lipid panel. Individuals taking the online questionnaire via Qualtrics were sent a link via email or scanned a QR code from poster recruitment. The link or code took them directly to the consent form which had to be answered with a "Yes" before beginning the questionnaire. For the subset of participants participating in the lipid panel, testing occurred at the Obesity Prevention Laboratory (OPL) at TCU following an overnight fast (12-14h). Knowledge, behaviors and attitudes

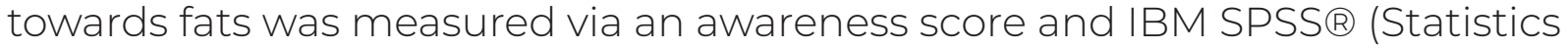
Version 25.0. Armonk, NY). Significance was set at $\mathrm{p}<0.05$. Further, blood lipids were measured via a lipid panel.

\section{Participants}

The recruitment process utilized TCU Announce, flyers, and in-person recruiting during class lectures and organization meetings Upon completion of recruiting participants, 107 women had completed the modified GNKQ; however, three of the responses had to be excluded due to not falling within the age range of 18 to 40 years old. Additionally, a subset of ten women consented to participate in the lipid panel. One of the women in the subset had to be excluded due to inability to obtain a blood draw on the day of her appointment. During the recruitment process, participants whom expressed interest in the lipid panel were emailed to discuss availability; once confirmed, participants were instructed to fast for twelve to fourteen hours before their scheduled lab visit. Lab visits were scheduled for Friday November 16 from 7:00am to 9:00am and Wednesday November 28 from 7:00am to 9:00am. Participants were scheduled in 15-minute intervals and instructed to arrive to the second floor of the Annie Richardson Bass Building at TCU at their respected time. 


\section{Study Protocol}

During the recruitment process, after speaking at class lectures or organization meetings, the professor or president sent out an email with the Qualtrics link included to complete the questionnaire. The link took the individual directly to the consent form, and once answered the questionnaire would begin. The GNKQ questionnaire consisted of seventy questions broken down into three categories: 34 knowledge questions, 21 behavior questions, and 15 awareness questions. The knowledge-based questions were the only questions used for scoring; however, researchers utilized all questions in the statistical analysis.

The subset of participants would complete the questionnaire the morning of their appointment. Researchers greeted participants upon arriving to the Bass building and escorted them to an exam room where anthropometrics, $\mathrm{BMI}$, and hip/waist ratio information were recorded. Participants were then escorted across the hall by a researcher for a $10 \mathrm{ml}$ fasting blood draw collection. Blood samples were centrifuged at $4^{\circ} \mathrm{C}$ at 3,000rpm for 15 minutes and chilled in a cooler immediately following centrifuging. Once the blood draw was complete, researchers escorted participants to a waiting area where they could complete the 15-minute GNKQ. Once participants completed the questionnaire, researchers escorted them out of the building. At the end of all blood draws, researchers transferred the blood samples to Any Lab Test Now where lipid panels were completed.

\section{Statistical Analyses}

The IBM SPSS version 24 statistical package will be used for all data analysis (SPSS Inc., Armonk, NY). Descriptive statistics including mean, range, and standard deviation will be calculated for all blood lipid variables. All values will be expressed as mean \pm standard error of the mean (SEM) unless otherwise indicated. Frequency and percentages will be computed to describe the characteristics of the sample. Correlations between anthropometric data, GNKQ responses, and blood lipid levels. The questionnaire will be completed, and results recorded through Qualtrics Survey. Statistical significance is set at $p<0.05$. 


\section{CHAPTER IV RESULTS}

\section{Participants}

The mean age (years) of participants who completed the survey was $25 \pm$ 7.3. Participants reported a mean weight $(\mathrm{kg})$ of $61.1 \pm 31.6$. Participants recorded their classification and major as well; results are shown in Figures 1 and 2.

\section{GNKQ - Knowledge-based Questions}

Researchers assigned participants with an awareness score based on the number of knowledge-based questions answered correctly. When researchers analyzed the score criteria, participants who answered 0 to 11 correctly, received a score of poor awareness of lipids in the diet. Participants who answered 12 to 23 questions correctly received a score of moderate awareness of lipids in the diet, and participants who answered 24 questions correctly received a score of strong awareness of lipids in the diet. The mean score was a 23.7, which equates to $69.7 \%$ of the questions answered correctly. Only one individual classified as a senior Nutrition major answered all 34/34 questions correctly. Results of the 104 participants' awareness score is in Figure 3. Table 5 shows an example of the type of knowledge-based questions asked and highlights the reported percentage of individuals who answered them correctly.

\section{GNKQ - Behavior-based Questions}

Participants were asked behavior-based questions to get an idea of foods consumed in the typical week. Olive oil was the most reported fat used when cooking at home with $48.6 \%$ of participants utilizing it; while the second most reported fat was butter with $27.1 \%$ of participants. Participants were additionally asked a series of questions regarding how often they eat a particular food item; the results are in Table 4, which displays the percentage of participants who reported eating the food item multiple days of the week. 


\section{Table 4: GNKQ PARTICIPANT DIET BEHAVIORS}

\begin{tabular}{lc}
\hline \multicolumn{1}{c}{ Often eat... } & Consumed multiple times a week \\
\hline Meat & $74.8 \%$ \\
\hline Regular-Fat Cheese & $73.8 \%$ \\
\hline Fried Foods & $50.4 \%$ \\
\hline Regular-Fat Chips & $72.0 \%$ \\
\hline Regular-Fat Ice Cream & $42.9 \%$ \\
\hline Processed Meats & $41.5 \%$ \\
\hline
\end{tabular}

GNKQ - Attitude-based Questions

GNKQ - Attitude-based Questions

A series of questions in the GNKQ allowed researchers to understand the attitude towards fats and whether or not the participants knew of the different types of fats in the diet. One question contained a series of parts giving a type of fat and allowing the participant to answer "yes" "no" or "not sure;" these responses are in Table 6. When individuals were asked how concerned they were with their fat consumption, $40.2 \%$ stated they were concerned, while the majority, 59.8\%, claimed the opposite. 


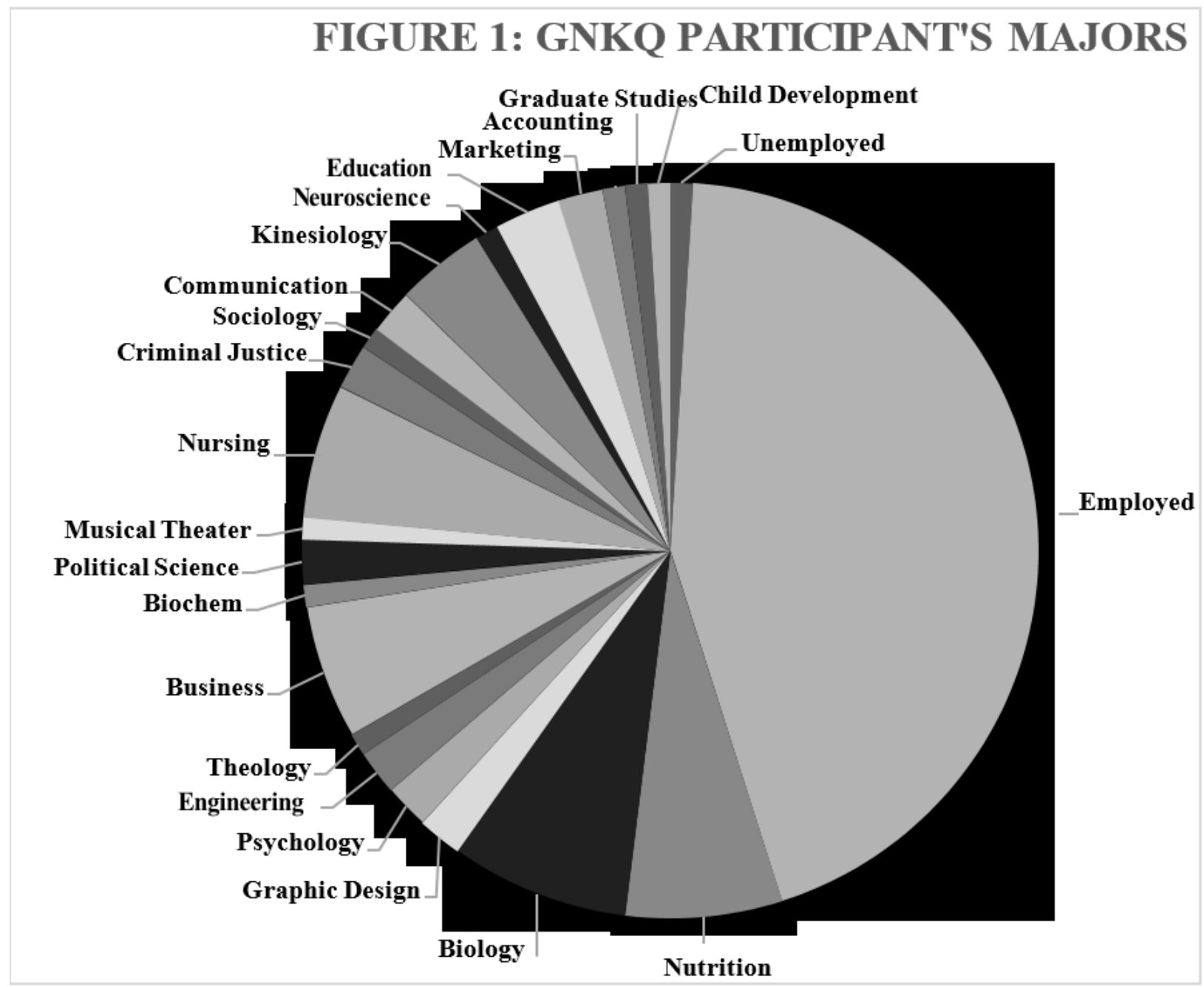


Figure 2: GNKQ PARTICIPANT'S CLASSIFICATIONS

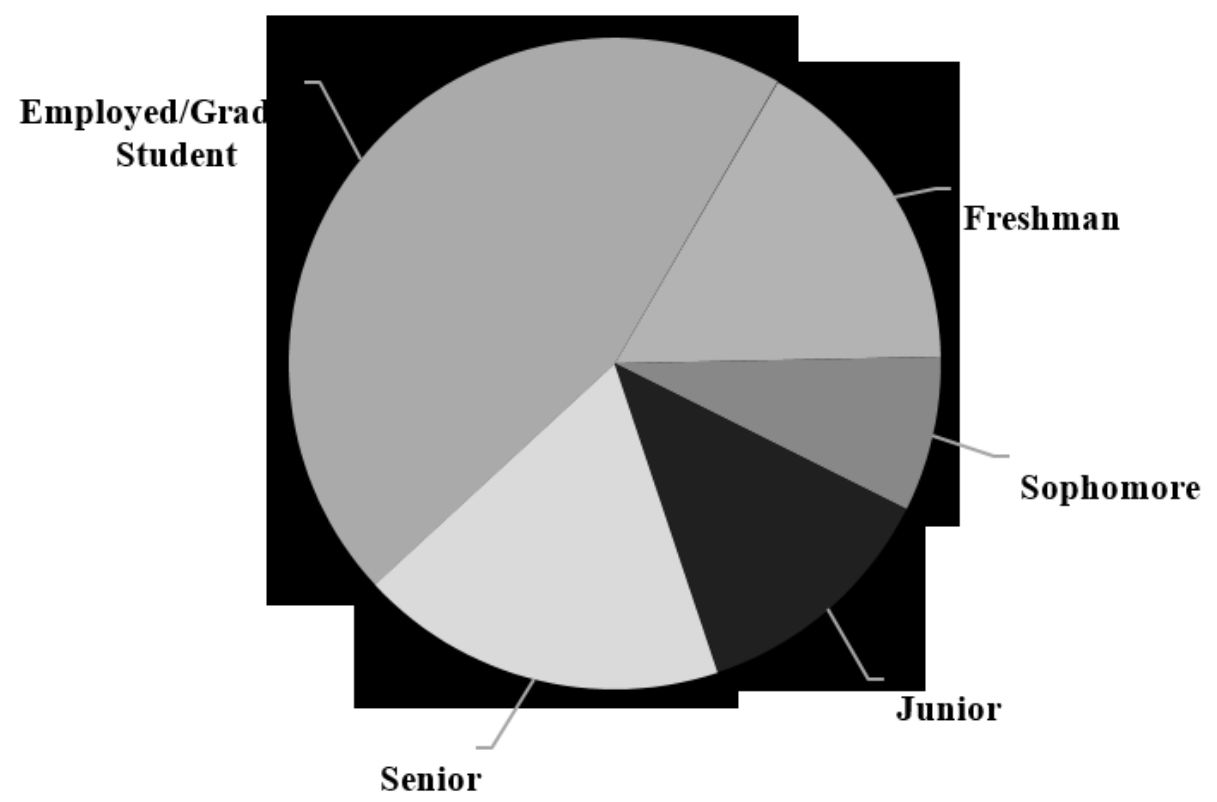

\section{Figure 3: GNKQ Awareness Scores}

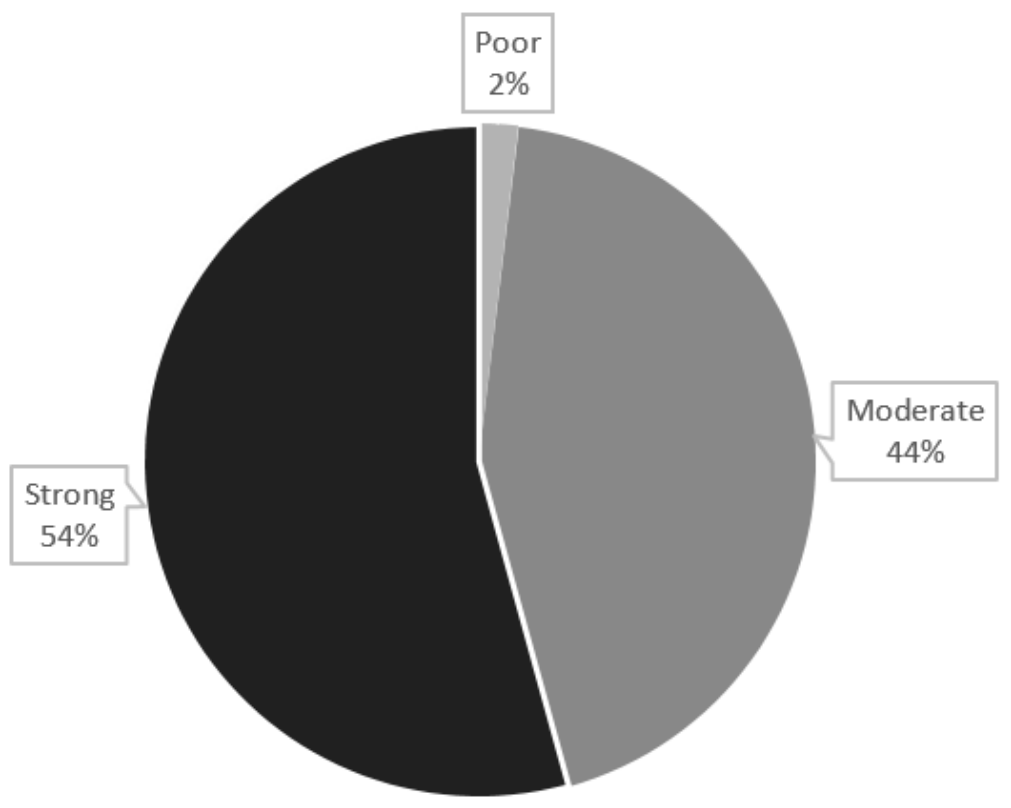


Table 5: GNKQ AWARENESS VS. KNOWLEDGE

\begin{tabular}{lcc}
\hline & Reported Awareness & Correctly Identified \\
\hline Saturated Fat & $99.1 \%$ & $44.9 \%$ \\
\hline Trans Fat & $99.1 \%$ & $74.8 \%$ \\
\hline Monounsaturated Fat & $73.6 \%$ & $25.2 \%$ \\
\hline Polyunsaturated Fat & $73.8 \%$ & $30.8 \%$ \\
\hline Fish Oil & $95.3 \%$ & - \\
\hline Omega 3 & $93.4 \%$ & - \\
\hline Omega 6 & $68.6 \%$ & - \\
\hline Animal Fat & $84.1 \%$ & - \\
\hline Vegetable Oil & $99.1 \%$ & - \\
\hline Hydrogenated Oil & $65.4 \%$ & - \\
\hline Partially Hydrogenated Oil & $58.5 \%$ & - \\
\hline Tropical Oil & $35.6 \%$ & - \\
\hline Steric Acid & $26.7 \%$ & - \\
\hline
\end{tabular}


Table 6: SURVEY RESPONSE FREQUENCIES

\begin{tabular}{lcc}
\hline & Correctly Identified & Answer \\
\hline $\begin{array}{l}\text { Experts recommend which } \\
\text { fat to be cut down in diets }\end{array}$ & $93.5 \%$ & Saturated Fat \\
\hline $\begin{array}{l}\text { To maintain a healthy diet } \\
\text { people should cut fat out } \\
\text { completely }\end{array}$ & $97.2 \%$ & FALSE \\
\hline $\begin{array}{l}\text { Are there any health } \\
\text { problems related to the } \\
\text { amount of fat in the diet }\end{array}$ & $63.6 \%$ & TRUE \\
\hline $\begin{array}{l}\text { Some foods contain a lot of } \\
\text { fat but no cholesterol }\end{array}$ & $35.5 \%$ & TRUE \\
\hline $\begin{array}{l}\text { Experts recommend eat more } \\
\text { or less meat }\end{array}$ & $59.8 \%$ & LESS \\
\hline $\begin{array}{l}\text { Which cooking method } \\
\text { requires fat to be added }\end{array}$ & $68.2 \%$ & Sautéing \\
\hline
\end{tabular}

Subset: Lipid Panel Participants

A subset of ten participants were recruited for a lipid panel; one participant was excluded due to the inability to obtain a blood draw. The mean age of the subset was $19.6 \pm 1.5$ years.

The mean anthropometric measurements obtained are in Table 7. Majors and classifications are presented in Figure 4. In this subset, effort was made to include various majors. The same awareness scoring methods were used for the lipid panel participants, and the results are recorded in Figure 5.

Mean results of the lipid panel compared to normal levels are represented in Figure 7. All participants had HDL above the recommended amounts, >39 milligrams per deciliter ( $\mathrm{mg} / \mathrm{dL}$ ); and all participants had a risk ratio below the recommended amounts, <3.22. Two participants had LDL levels above recommended limits, $<100 \mathrm{mg} / \mathrm{dL}$, and one participant had total cholesterol above recommended amounts, $<200 \mathrm{mg} / \mathrm{dL}$. All participants had triglyceride levels within normal limits, $<150 \mathrm{mg} / \mathrm{dL}$. 


\section{Correlations}

Correlations for lipid levels/body composition versus GNKQ responses are displayed in Table 8. A significant, positive correlation was found between HDL level of the participants, and how often they reported eating the skin or fat on meat ( $r=0.803, p=0.009$ ), with the higher the HDL level the more the individuals reported consuming the skin or fat on meat. Additionally, a significant positive correlation was discovered between the higher an individual's LDL level and the more times participants reported eating fried foods $(r=0.696, p=0.037)$.

A significant positive correlation was present between the larger the individuals' weight, the higher their risk ratio $(r=0.846, p=0.004)$. Risk ratio is the ratio of (HDL/LDL) represented as a number to show the risk of developing conditions from high LDL levels like CVD. There was a significant positive correlation between $\mathrm{BMI}$ and waist-hip ratio $(r=0.740, p=0.023)$ as well. The higher the individuals' triglyceride levels, the larger their waist-hip ratio ( $r=0.757$, $\mathrm{p}=0.018)$. Waist-hip ratio was positively correlated both with age $(r=0.832$, $\mathrm{p}=0.005)$ and how often an individual reported eating fried food $(r=0.733$, $\mathrm{p}=0.025)$.

Table 7: LIPID PANEL PARTICIPANT CHARACTERISTICS

\begin{tabular}{lc}
\hline Height $(\mathrm{cm})$ & $164.6 \pm 6.1$ \\
\hline Weight $(\mathrm{kg})$ & $61.1 \mathrm{~kg} \pm 10.8$ \\
\hline BMI & $22.5 \pm 3.7$ \\
\hline Waist Measurement $(\mathrm{cm})$ & $70.82 \pm 0.43$ \\
\hline Hip Measurement $(\mathrm{cm})$ & $95.71 \pm 0.59$ \\
\hline Waist/Hip Ratio & $0.74 \pm 0.01$ \\
\hline
\end{tabular}


Table 8: CORRELATIONS BETWEEN LIPID PANEL RESULTS AND GNKQ RESPONSES

\begin{tabular}{|c|c|c|c|c|c|c|c|c|c|c|c|}
\hline & $\begin{array}{l}\text { Often Eat } \\
\text { Meat }\end{array}$ & $\begin{array}{l}\text { Often Eat Fried } \\
\text { Foods }\end{array}$ & $\begin{array}{l}\text { Often Eat Skin or Fat } \\
\text { on Meat }\end{array}$ & Age & BMI & Weight & $\begin{array}{l}\text { Waist-Hip- } \\
\text { Ratio }\end{array}$ & HDL & LDL & Triglycerides & Risk Ratio \\
\hline Often Eat Meat & & $0.432 ; \mathrm{p}=0.246$ & $0.806^{* *} ; \mathrm{p}=0.009$ & $-0.742 ; p=0.200$ & $-0.258 ; p=0.503$ & $-0.483 ; p=0.188$ & $-0.237 ; p=0.539$ & $0.767^{*} ; \mathrm{p}=0.016$ & $0.205 ; p=0.597$ & $0.115 ; p=0.769$ & $-0.441 ; p=0.234$ \\
\hline $\begin{array}{l}\text { Often Eat Fried } \\
\text { Foods }\end{array}$ & & & $0.432 ; \mathrm{p}=0.246$ & $\begin{array}{l}-0.742^{8} \\
\mathrm{p}=0.022\end{array}$ & $-0.443 ; p=0.232$ & $-0.489 ; \mathrm{p}=0.181$ & $-0.733^{*} ; \mathrm{p}=0.025$ & $-0.011 ; \mathrm{p}=0.977$ & $-0.696^{*} ; \mathrm{p}=0.037$ & $\begin{array}{l}-0.345 \\
\mathrm{p}=0.363\end{array}$ & $-0.689 * ; \mathrm{p}=0.40$ \\
\hline $\begin{array}{l}\text { Often Eat Skin or Fat } \\
\text { on Meat }\end{array}$ & & & & $-0.478 ; p=0.193$ & $0.038 ; p=0.923$ & $-0.187 ; p=0.630$ & $-0.191 ; \mathrm{p}=0.622$ & $\begin{array}{l}0.803^{* * 8} \\
p=0.009\end{array}$ & $0.245 ; \mathrm{p}=0.525$ & $0.099 ; \mathrm{p}=0.800$ & $-0.361 ; p=0.340$ \\
\hline Age & & & & & $0.118 ; p=0.763$ & $0.291 ; \mathrm{p}=0.447$ & $\begin{array}{l}0.832 * * ; \\
p=0.005\end{array}$ & $-0.275 ; \mathrm{p}=0.473$ & $0.410 ; \mathrm{p}=0.273$ & $0.478 ; p=0.194$ & $0.633 ; \mathrm{p}=0.067$ \\
\hline BMI & & & & & & $\begin{array}{l}0.907 * * ; \\
p=0.001\end{array}$ & $0.266 ; p=0.489$ & $-0.231 ; \mathrm{p}=0.550$ & $0.448 ; \mathrm{p}=0.226$ & $0.473 ; p=0.198$ & $0.740^{*} ; \mathrm{p}=0.023$ \\
\hline Weight & & & & & & & $0.350 ; \mathrm{p}=0.356$ & $-0.413 ; \mathrm{p}=0.269$ & $0.413 ; \mathrm{p}=0.269$ & $0.358 ; p=0.344$ & $\begin{array}{l}0.846^{* * *} \\
p=0.004\end{array}$ \\
\hline Waist-Hip Ratio & & & & & & & & $-0.098 ; \mathrm{p}=0.802$ & $0.548 ; \mathrm{p}=0.127$ & $\begin{array}{l}0.757 * ; \\
p=0.018\end{array}$ & $0.642 ; p=0.062$ \\
\hline $\mathrm{HDL}$ & & & & & & & & & $0.466 ; p=0.206$ & $\begin{array}{l}-0.103 ; \\
p=0.792\end{array}$ & $-0.371 ; \mathrm{p}=0.326$ \\
\hline LDL & & & & & & & & & & $0.424 ; p=0.255$ & $0.639 ; p=0.064$ \\
\hline Triglycerides & & & & & & & & & & & $0.564 ; p=0.114$ \\
\hline
\end{tabular}

*Table represents correlations for the subset of nine individuals between lipid panel results and survey responses. Body Mass Index (BMI), High Density Lipoprotein (HDL), Low Density Lipoprotein (LDL), and Risk Ratio (HDL/LDL). * denotes a significance at the 0.05 level (2-tailed). ${ }^{* *}$ denotes a significance at the 0.01 level (2-tailed). 
Figure 4: LIPID PANEL PARTICIPANT'S MAJORS
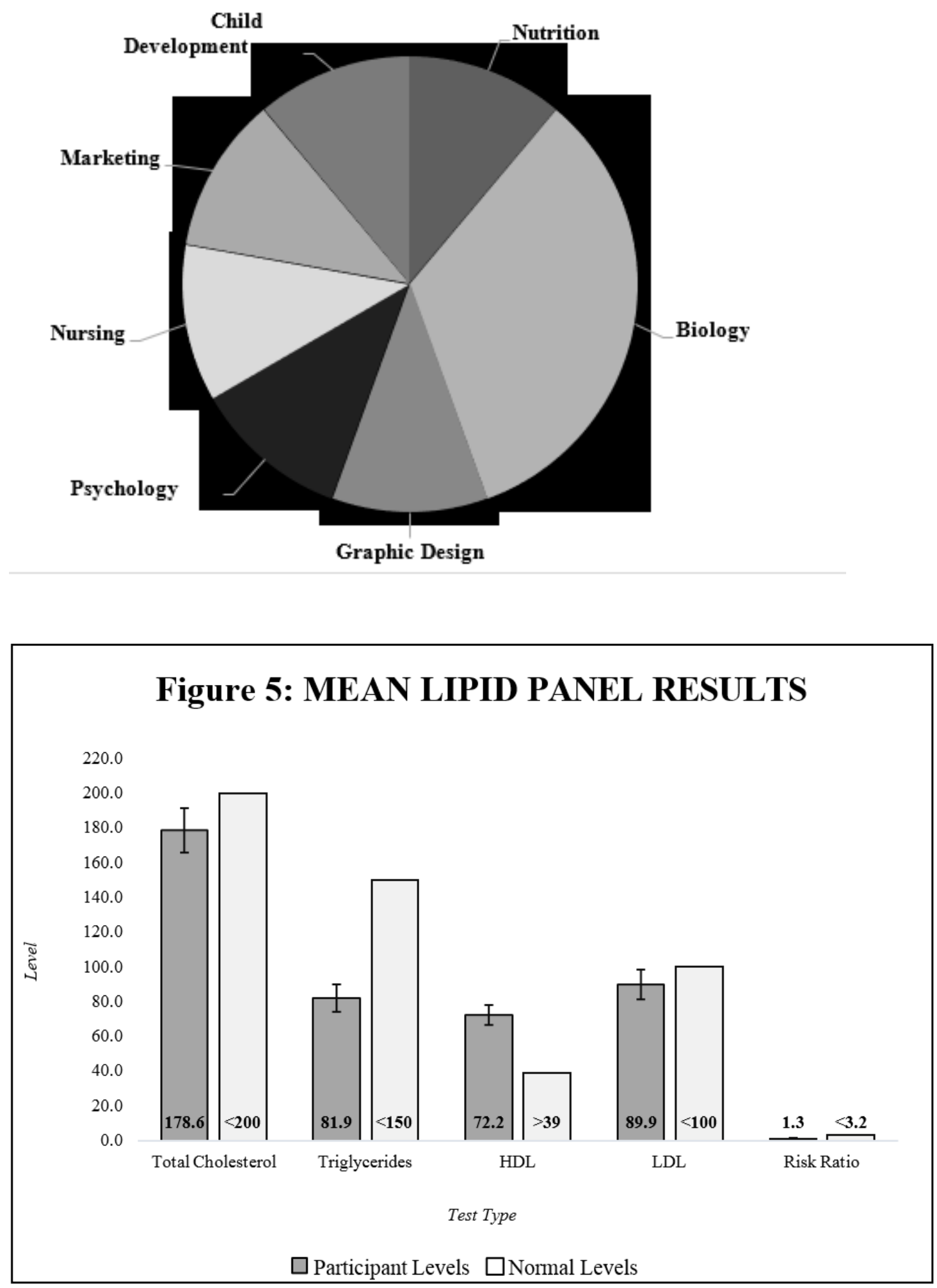


\section{CHAPTER V DISCUSSION}

The aim of this study was two-fold. We aimed to determine: 1) knowledge, attitudes, and behaviors of dietary fats using the modified GNKQ; and 2) correlations between anthropometric data, GNKQ responses, and blood lipid levels. During review of the GNKQ responses, we found a common trend of women reporting awareness of specific types of fats in the survey; however, when asked a knowledge-based question to identify where this specific fat was located, the women were unable to correctly identify where it was located, representing a nutrition education gap.

When looking at frequencies further, when participants were asked general questions regarding what experts recommend in the diet to prevent heart disease as in more or less salty foods, fat, sugar, fruits/vegetables, and fiber; greater than $75 \%$ answered each of these questions correctly. The majority of participants know what experts recommend as a healthy diet to prevent heart disease. This is another theme found by Gillian Anne Hendrie and others where $90 \%$ or more of the participants taking part in a nutrition knowledge study were aware of the expert recommendations to eat more fruits and vegetables 14 . Furthermore, our research found that less than $50 \%$ of participants know the specific serving amounts regarding how much fish to consume each week, along with other food serving specifics. This was a common theme seen in Gillian Anne Hendrie et al.'s study as well where only $56 \%$ of participants knew the recommended number of fruits and vegetables to consume each day 74 . Participants know the surface level questions regarding what to eat; however, do not know the detailed questions regarding serving sizes. The mean score for the GNKQ was a $69.7 \%$ in our study. We found no significance when comparing the individual awareness scores to age or income level; however,

in Emily R McLeod and others' study, they found quite the opposite. In mothers of low, middle, and upper socioeconomic status there was a significant positive correlation between the higher socioeconomic status, the higher the general nutrition knowledge score was15.

When researchers analyzed the lipid panel results and anthropometric data, a strong positive correlation was discovered between an individual's triglyceride levels and their waist- hip ratio. This positive correlation was also seen in Dr. Mabel Yap's study where high serum triglyceride levels were higher in individuals with higher waist-hip ratios76. High waist-hip ratios and high triglyceride levels are both risk factors for obesity and CVD; this correlation is not surprising when considering the effect both risk factors have on overall health.

A few limitations in this research study warrant discussion. The modified GNKQ used contained questions that were subjective to the individual, including the answer choices of "often," "sometimes," and "rarely/never." What is 
"often" to one individual may not be "often" to another; thus, the frequency of food consumption reported may not have been accurate for each individual or consistent for all individuals. Additionally, this research project, originally joined with another, consisted of solely female participants ranging in age from 18 to 40 years old, thus why the same inclusion criteria is set on our participants; this limited the research from exploring male populations and a wider age range. Due to timing and scheduling constraints, the sample of women who completed the lipid panel were all college students ranging in age of 18 to 22 years old, a small age range to represent the comparison of knowledge and lipid panel results across all life spans. Furthermore, due to funding and time limitations, the small subset of lipid participants does not provide sufficient results. We recommend further research with a larger subset of males and females to complete surveys and lipid panels.

\section{CHAPTER VI CONCLUSIONS}

The present findings indicate that individuals are aware of the different types of FAs and fats in the diet; however, the majority lacks the ability to correctly identify where these fats are located. Additionally, the findings show that individuals are aware of the diets that experts recommend to prevent heart disease. When individuals were asked higher-level thinking questions regarding the impact of diet on disease and cholesterol levels, individuals were not able to answer them correctly. This is important information for future nutrition education and public health efforts to have in order to bridge knowledge gaps. Despite the present findings of the subset participants with cholesterol levels in normal limits, this is not indicative of the population as a whole. More large-scale research should be done including both males and females who complete both a questionnaire and lipid panel. 


\section{Reference List}

Abraham SB, Rubino D, Sinaii N, Ramsey S, Nieman LK. Cortisol, obesity, and the metabolic syndrome: A cross-sectional study of obese subjects and review of the literature. 2012:105-17. https://doi:0.1002/oby.20083.

Aquilani R, Parisi U, Bigoni N, et al. School teachers can effectively manage primary prevention of adult cardiovascular disease. The Stradella Project. Preventive Medicine. 2007;45(4):290-294. https://doi:10.1016/j.ypmed.2007.06.018.

Boyle M. Personal Nutrition. 9th edition. Boston, MA: Cengage Learning. 2014:133.

Brenna JT, Crawford MA, Elmadfa I, et al. Interim summary of conclusions and dietary recommendations on total \& fatty acids. Food and Agriculture Organ/World Health Organ. 2008;1:1-12. www.fao.org/ag/agn/nutrition/docs/ Fats\%20and\%20Fatty\%20Acids\%20Summaryfin.pdf

Catapano AL, Wiklund O. Think Again About Cholesterol Survey. Atherosclerosis (Supplements). 2015;20:1-5. https://doi:10.1016/S1567-5688(15)30011-8.

Deurenberg-Yap M, Yian T, Kai C, Deurenberg P, and Staveren W. Manifestation of cardiovascular risk factors at low levels of body mass index and waist-tohip ratio in Singaporean Chinese. Asia Pacific Journal of Clinical Nutrition. 2002; 8(3). https://doi.org/10.1046/j.1440-6047.1999.00091. 2002.01.18.

Hendrie G, Coveney J, and Cox D. Exploring nutrition knowledge and the demographic variation in knowledge levels in an Australian community sample. Public Health Nutrition. 2008;(12), 1365-71. https://doi:10.1017/ S1368980008003042. 2018.08.01.

High Cholesterol. Mayoclinic.org. https://www.mayoclinic.org/diseases-conditions/high-blood-cholesterol/symptoms-causes/syc-20350800. Accessed April 15, 2018.

Making sense of cholesterol tests. Harvardhealth.edu. https://www.health.harvard.edu/heart-health/making-sense-of-cholesterol-tests. Published February 2005. Updated April 2, 2018. Accessed April 15, 2018. 
McLeod E, Campbell K, and Hesketh K. Nutrition knowledge: a mediator between socioeconomic position and diet quality in Austrailain first-time mothers. Journal of the American Dietetic Association. 2011;117(5),696-704. https://doi.org/10.1016/j.jada.2011.02.011.

Nelms M, Sucher KP, Lacey K. Nutrition Therapy and Pathophysiology. Cengage Learning. 2016:307-30.

Overweight and Obesity Statistics. National Institute of Diabetes and Digestive and Kidney Diseases(niddk.nih.gov). https://www.niddk.nih.gov/health-information/health- statistics/overweight-obesity. Published August 2017. Accessed April 18, 2018.

Pinheiro MM, Wilson T. Dietary fat: the good, the bad, and the ugly. SpringerLink. 2017;1:241-247. https://doi:10.1007/978-3-319-49929-1_24.

Stevenson JL, Miller MK, Skillman HE, Paton CM, Cooper JA. A PUFA-rich diet improves fat oxidation following saturated fat-rich meal. Eur J of Nutr. 2017;56(5):1845- 1857. https://doi:10.1007/s00394-016-1226-9.

Stewart J, Manmathan G, Wilkinson P. Primary prevention of cardiovascular disease: a review of contemporary guidance and literature. London Cardiovasc Soc. 2017;6:1-9. https://doi:10.1177/204800-40/66872/1.

Woolf SH, Aron L, Dubay L, Simon S, Zimmerman E, Luk K. How are income and wealth linked to health and longevity. Urban Inst. 2015:1-21. http://webarchive.urban.org/publications/2000178.html 